\title{
Evidence to date: ranibizumab and its potential in the treatment of retinopathy of prematurity
}

This article was published in the following Dove Press journal:

Eye and Brain

\section{Samir N Patel \\ Michael A Klufas}

The Retina Service of Wills Eye Hospital, Mid Atlantic Retina, Thomas Jefferson University, Philadelphia, PA, USA
Correspondence: Michael A Klufas

The Retina Service of Wills Eye Hospital, Thomas Jefferson University, 840 Walnut Street, Suite 1020, Philadelphia, PA 19107 USA

$\mathrm{Tel}+|80033| 6634$

$\mathrm{Fax}+12158259087$

Email mklufas@midatlanticretina.com

\begin{abstract}
Retinopathy of prematurity (ROP) is a leading and preventable cause of childhood blindness worldwide. Although laser photocoagulation remains the gold standard for treatment, the off-label use of anti-vascular endothelial growth factor (anti-VEGF) therapy to treat ROP, particularly posterior zone I disease, is increasing. Although initial studies on anti-VEGF therapy for ROP have focused on bevacizumab, recent studies have proposed that ranibizumab may be a safer and more effective alternative for use in this population. This review updates recent evidence regarding the use of ranibizumab in the management of ROP.
\end{abstract}

Keywords: retinopathy of prematurity, ranibizumab, bevacizumab, Lucentis, Avastin, vascular endothelial growth factor, RAINBOW, BEAT-ROP, anti-VEGF

\section{Introduction}

Retinopathy of prematurity (ROP) is a vasoproliferative disorder affecting the retinas of premature infants. The screening, treatment, and pathophysiologic understanding of ROP have dramatically evolved over the past four decades. Two landmark studies, Cryotherapy for ROP (CRYO-ROP) ${ }^{1}$ in 1988 and Early Treatment for ROP (ETROP) ${ }^{2}$ in 2004 have served as the stepping stones for establishing treatment guidelines with respect to threshold and prethreshold type 1 ROP.

Most recently, however, intravitreal anti-vascular endothelial growth factor (antiVEGF) agents for ROP have received much attention in the medical community as a potential alternative. Reported advantages of anti-VEGF pharmacotherapy over laser photocoagulation include decreased treatment time with less stress on the neonate, swift resolution of plus disease with prompt regression of ROP, potential of further retinal vascular development with no ablation of the peripheral avascular retina, lower risk of myopia, and improved treatment outcomes for zone I ROP or AP-ROP. ${ }^{3-5}$ AntiVEGF may also be the only treatment option in cases of media opacity or vitreous hemorrhage when an insufficient view is present for laser photocoagulation.

One of the largest anti-VEGF studies in ROP to date, the BEAT-ROP (Efficacy of Intravitreal Bevacizumab for Stage $3+$ Retinopathy of Prematurity) trial, ${ }^{5}$ found that bevacizumab (Avastin; Genentech Inc, South San Francisco, California, USA) can halt the progression of severe ROP, revert pathologic angiogenic changes, and induce the progression of physiologic intraretinal vasculature. Of note, BEAT-ROP was the first prospective study to investigate anti-VEGF use for ROP at time when the preponderance of literature was in the form of retrospective case reports and series. Although initial studies on anti-VEGF therapy for ROP have focused on bevacizumab, recent studies have proposed the use of ranibizumab (Lucentis; Genentech Inc, San Francisco, 
CA/Novartis Ophthalmics, Basel, Switzerland) for the treatment of ROP. In this article, we review the current evidence of ranibizumab for the management of ROP.

\section{Molecular structure and function}

Ranibizumab is a recombinant humanized monoclonal antibody fragment designed to bind and inhibit all biologically active isoforms of human VEGF. ${ }^{6}$ The VEGF family includes VEGF-A, VEGF-B, VEGF-C, VEGF-D, VEGF-E, and placental growth factor, among which the most important member is VEGF-A. ${ }^{7}$ VEGF-A is a dimeric, disulfide-bound glycoprotein that is specifically activated on endothelial cells and plays a key role in various processes such as inducing angiogenesis, accelerating the endothelial cell growth, promoting cell migration, and inhibiting apoptosis and tumor growth. At least six VEGF-A isoforms including $\mathrm{VEGF}_{121}, \mathrm{VEGF}_{145}$, $\mathrm{VEGF}_{165}, \mathrm{VEGF}_{183}, \mathrm{VEGF}_{189}$, and $\mathrm{VEGF}_{206}$ are produced by alternative splicing of the VEGF-A gene. ${ }^{8}$

Like ranibizumab, bevacizumab is another monoclonal antibody that binds and inhibits all isoforms of VEGF with a lower affinity (Table 1). Ranibizumab and bevacizumab locate in the receptor-binding region of VEGF and both antibodies target VEGF in a similar way. However, bevacizumab (149 kDa) and ranibizumab (48.39 kDa) have different molecular weights, mainly because ranibizumab does not contain a fragment crystallizable $(\mathrm{Fc})$ region. Figure 1 shows the molecular structure of ranibizumab that includes a heavy chain (antigen-binding fragment) and light chain. Furthermore, bevacizumab is produced in a eukaryotic cell line and is N-glycosylated in its Fc region, while ranibizumab is produced in prokaryotic $\mathrm{E}$. coli, and therefore it does not carry any glycosylation sites. Additionally, though both bevacizumab and ranibizumab are off-label treatments for ROP, for other ocular conditions, bevacizumab is only FDA approved for intravenous administration, whereas ranibizumab is approved and

Table I Differences in structure and function between ranibizumab and bevacizumab

\begin{tabular}{|l|l|l|}
\hline & Ranibizumab & Bevacizumab \\
\hline Molecule & Fab fragment & Full length antibody \\
Glycosylation & No & Yes \\
Fc & No & Yes \\
Molecular weight & $48.39 \mathrm{kDa}$ & $139 \mathrm{kDa}$ \\
Half-life & 3 days & $17-21$ days \\
\hline
\end{tabular}

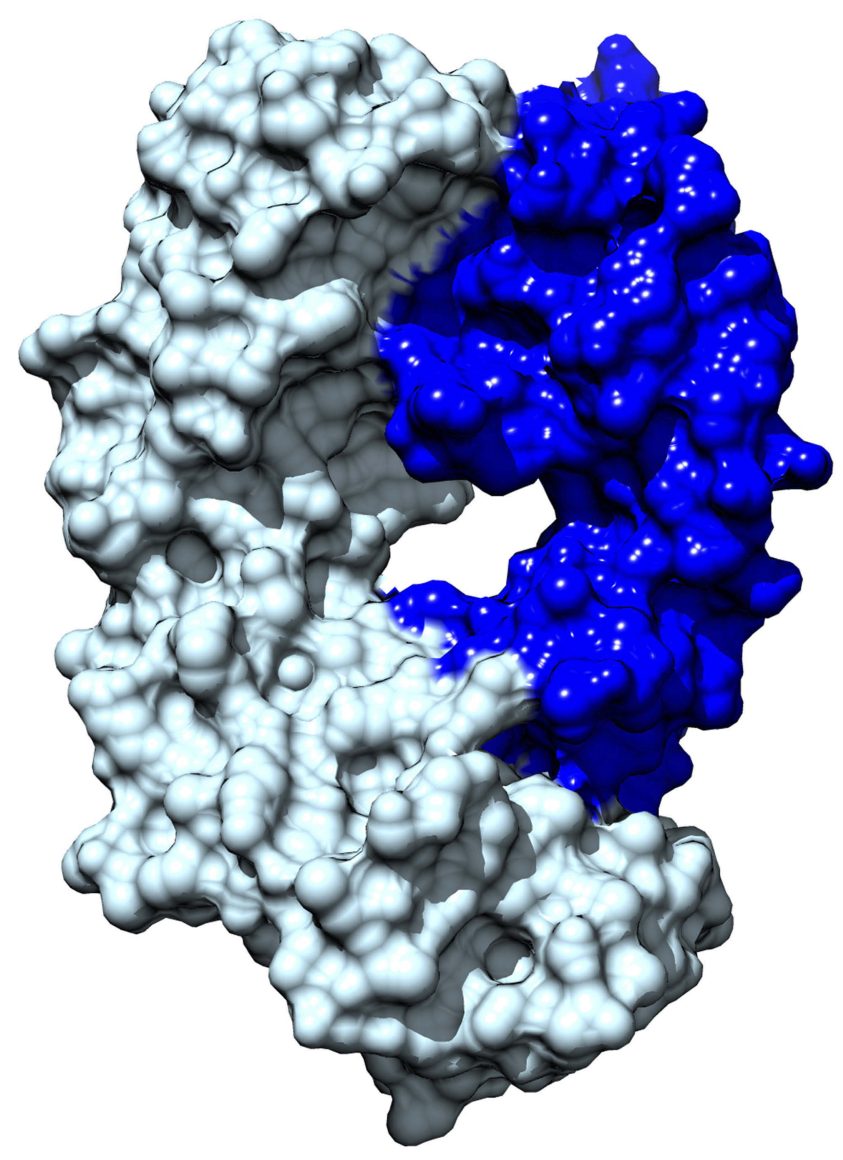

Figure I Molecular structure of ranibizumab. The heavy chain of the antigenbinding fragment is in dark blue and the light chain is in light blue. Permission to use this figure was obtained from Genetech.

formulated for intraocular administration. In clinical practice in the United States, bevacizumab is compounded for intraocular use from the intravenous formulation by compounding pharmacies.

In animal models assessing the vitreous pharmacokinetics of anti-VEGF medications, the vitreous half-life of 0.5 -mg intravitreal ranibizumab was 2.88 days, whereas the half-life of 1.25 -mg intravitreal bevacizumab was 4.32 days. ${ }^{9}$ Furthermore, no ranibizumab was detected in the serum or the fellow uninjected eye, but bevacizumab was detected in the serum and fellow uninjected eye. ${ }^{9}$ Systemic pharmacokinetics that reported that bevacizumab has a longer half-life of 17-21 days compared to 3 days for ranibizumab. ${ }^{10}$ These findings suggest that bevacizumab may potentially lead to more systemic absorption.

\section{Systemic safety data}

One of the most important barriers to widespread antiVEGF use in ROP is the lack of certainty regarding the 
systemic long-term effects of intravitreal anti-VEGF therapy in neonates. Nearly $66 \%$ of pediatrics and retina specialist in a recent survey reported a lack of certainty in the systemic effects of intravitreal anti-VEGF agents to treat ROP. ${ }^{11}$ The pharmacokinetic differences between bevacizumab and ranibizumab may be particularly important for safety considerations in premature infants undergoing organogenesis. VEGF is important to kidney, brain, and lung development, as well as to retinal and neuronal health. ${ }^{12}$ Inhibiting VEGF thus raises concerns about developing organs in the premature infant.

In adults with exudative age-related macular degeneration, Carneiro et $\mathrm{al}^{13}$ showed bevacizumab significantly reduced VEGF plasma levels until 28 days after intravitreal injection, whereas ranibizumab did not achieve significant plasma VEGF reduction at the same time-point. Sato et $\mathrm{al}^{14}$ showed VEGF level significantly decreased 1 week after intravitreal bevacizumab in ROP infants, and the VEGF level had a significant negative correlation to serum bevacizumab level. Their findings suggest that bevacizumab escapes from the vitreous into the systemic circulation and reduces the serum VEGF concentrations in ROP infants after the intravitreal bevacizumab. Two additional studies found that intravitreal bevacizumab results in low VEGF serum levels for at least 8 weeks ${ }^{15}$ and up to 12 weeks after the treatment. ${ }^{16}$ In contrast, when using ranibizumab, one study showed that intravitreal ranibizumab can lower serum levels of VEGF by 1 day after treatment, but this effect is short lived and serum VEGF levels recover to baseline within 1 week of treatment. ${ }^{17}$ Furthermore, in the CARE-ROP study (Comparing Alternative Ranibizumab Dosages for Safety and Efficacy in Retinopathy of Prematurity), plasma VEGF levels were not altered in 9 infants receiving $0.12 \mathrm{mg}$ or in 7 infants receiving $0.20 \mathrm{mg}$ of ranibizumab. ${ }^{18}$ However, Wong et $\mathrm{al},{ }^{19}$ in a case series, reported paradoxical improvement in both eyes of a patient undergoing unilateral intravitreal ranibizumab injection, which supports the notion that decreased systemic VEGF levels from unilateral injections may have clinically relevant effects.

Whether there are neurodevelopmental complications related to anti-VEGF treatment in neonates remains to be seen. No level I studies have addressed the long-term ocular, visual, systemic, or neurodevelopmental effects of intraocular anti-VEGF treatment. This is partly due to the need for a very large patient population and very long follow-up for such a study to be powered correctly to sort out confounding variables given that treatment-requiring ROP is known to be associated with comorbidities and poor neurodevelopmental outcomes. Despite these limitations, the current evidence is variable regarding neurodevelopmental outcomes in infants with ROP receiving anti-VEGF therapy. Morin et $\mathrm{al}^{20}$ noted that, in 125 infants treated for ROP, the odds of having a severe neurodevelopmental disability (severe cerebral palsy, hearing aids, or bilateral blindness) were 3.1 times higher with bevacizumab versus laser treatment. However, the authors acknowledged the study was limited by being retrospective, nonrandomized and because infants treated with bevacizumab tended to be sicker with more severe health problems. In contrast, Araz-Ersan et $\mathrm{al}^{21}$ evaluated series of 13 infants treated with combination intravitreal bevacizumab and laser therapy for ROP, compared with an age-matched control group of children who had received laser treatment for ROP, and found no difference in the mean cognitive, language, or motor scores based on the Bayley Scales of Infant Development (BSID) test. Lien et $\mathrm{al}^{22}$ reported no difference in mental or psychomotor impairment when comparing bevacizumab monotherapy to laser monotherapy, but patients with combination therapy of laser and bevacizumab had higher incidence of mental or psychomotor impairment.

\section{Current literature on ranibizumab for ROP}

A 2017 report by the American Academy of Ophthalmology on the use of anti-VEGFs for the primary treatment of ROP noted no level I evidence on the subject. ${ }^{3}$ A recent Cochrane review in 2018 concluded that there was insufficient evidence to provide a strong conclusion for the routine use of intravitreal anti-VEGFs in clinical practice. ${ }^{23}$ To date, eight head-to-head studies ${ }^{19,24-31}$ have been published comparing intravitreal ranibizumab to intravitreal bevacizumab and/or conventional laser photocoagulation for ROP and three retrospective studies ${ }^{32-34}$ with at least 100 eyes have been published on outcomes of infants treated intravitreal ranibizumab monotherapy (Table 2).

In 2017, Zhang et $\mathrm{al}^{24}$ reported outcomes of a prospective randomized clinical trial for 50 infants who had bilateral type 1 ROP in zone II who were randomized to receive either intravitreal ranibizumab $(0.3 \mathrm{mg}$ in 0.03 $\mathrm{mL})$ or diode $(810 \mathrm{~nm})$ laser photocoagulation. No information was available on randomization administration and/or masking. The main outcomes assessed were regression of ROP and plus disease, recurrence requiring treatment, and complications. In the ranibizumab group, 26 of $50(50 \%)$ eyes demonstrated recurrence and underwent laser photocoagulation with a mean interval to retreatment of $12.6 \pm 7.9$ weeks. In the laser group, 2 of $40(4 \%)$ eyes 







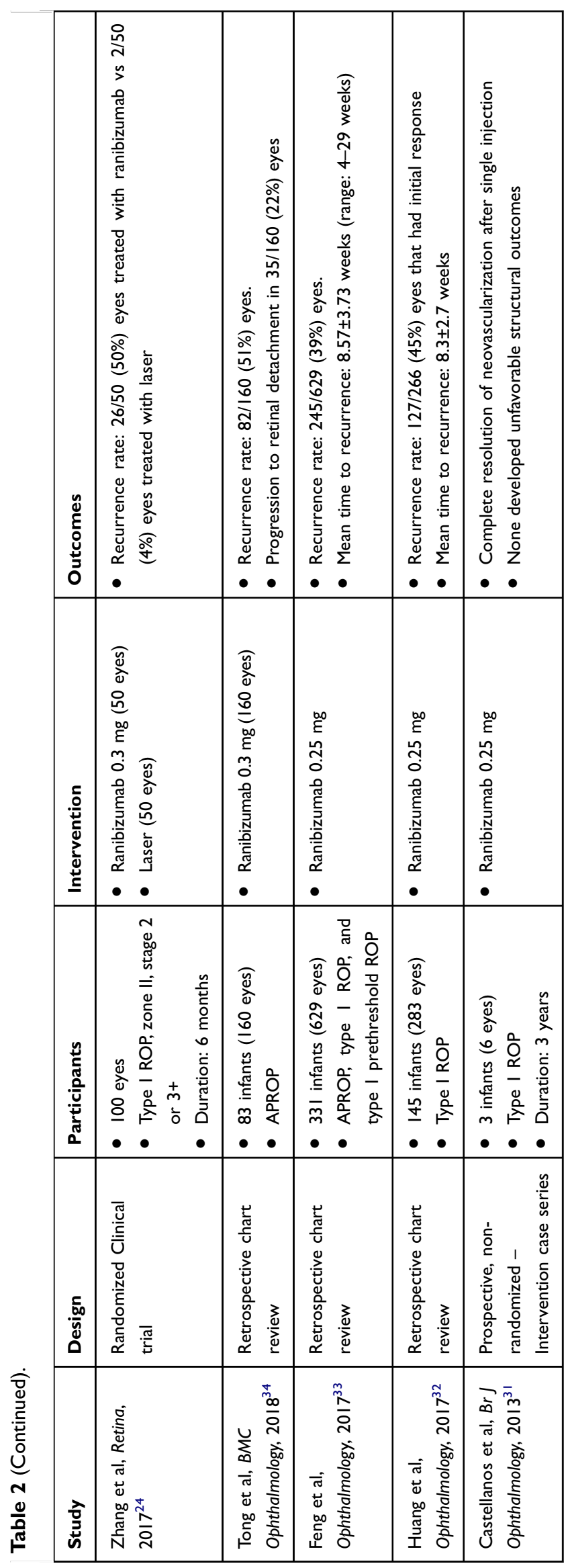


did not show an initial response and subsequently received ranibizumab injections 1 week after the laser treatment.

In 2017 , Gunay et $\mathrm{al}^{25}$ reported outcomes of a retrospective review of 134 infants (264 eyes) with type 1 ROP or aggressive posterior ROP at 2 large referral centers in Turkey who received either intravitreal bevacizumab (55 infants; dose, $0.625 \mathrm{mg}$ ), intravitreal ranibizumab (22 infants; dose, $0.25 \mathrm{mg}$ ), or diode laser photocoagulation (57 infants). Main outcome measures assessed were regression of ROP, recurrence profile, complications after each treatment modality, and indications for retreatment. All eyes showed an initial response to treatment, but recurrence of ROP was seen in 3 of 55 infants $(5.5 \%)$ treated with intravitreal bevacizumab, 11 of $22(50 \%)$ infants treated with intravitreal ranibizumab, and 1 of 57 infants $(1.7 \%)$ treated with laser photocoagulation. All infants with recurrence in the bevacizumab group required bilateral retreatment, but only 3 of the $11(3 \%)$ with recurrence in the ranibizumab group required bilateral retreatment. At the last follow-up, the prevalence of emmetropia was significantly higher in the groups that received antiVEGF therapy compared with the laser-treated group (50.9\% of the bevacizumab group, $45.5 \%$ of the ranibizumab group, and $16.3 \%$ of the laser group).

In 2016, Alyamac et $\mathrm{al}^{26}$ reported outcomes of a retrospective review of 45 infants (90 eyes) with type 1 ROP in zone I or posterior zone II who received either intravitreal bevacizumab (44 eyes; dose, $0.625 \mathrm{mg}$ ) or intravitreal ranibizumab (46 eyes; dose, $0.25 \mathrm{mg}$ ). Main outcome measures were rates of retinal vascularization. The mean time to complete vascularization was $55.93 \pm 4.13$ weeks PMA in the bevacizumab group and 56.30 \pm 4.30 weeks PMA in ranibizumab group. Recurrence was seen in 14 of $23(61 \%)$ infants treated with ranibizumab and 6 of 22 $(10 \%)$ infants treated with bevacizumab. Two of $6(33 \%)$ infants with recurrence in the bevacizumab group required diode laser photocoagulation as additional treatment at 43 weeks PMA, whereas 2 of 14 (14\%) infants with recurrence in the ranibizumab group required diode laser photocoagulation as additional treatment at 42.5 weeks PMA.

In 2015 , Chen et $\mathrm{al}^{27}$ reported outcomes of a retrospective review of 37 infants (72 eyes) with type 1 ROP who received either intravitreal bevacizumab (41 eyes; dose, $0.625 \mathrm{mg}$ ) or intravitreal ranibizumab (31 eyes; dose, $0.25 \mathrm{mg}$ ). Main outcome measures assessed were recurrence of ROP and refractive errors at a corrected age of 1 year. No recurrence of ROP occurred in either group if the patients initially responded to either bevacizumab or ranibizumab. All but one eye in the bevacizumab group had retinal neovascularization and plus disease regression after anti-VEGF treatment that required diode laser photocoagulation. There was no difference in mean refractive error between bevacizumab or ranibizumab. However, there were 6 of 41 eyes $(14.6 \%)$ in the bevacizumab group with high myopia (spherical equivalent $\leq-5.0$ diopters) compared to 0 of 31 eyes $(0 \%)$ in the ranibizumab group.

In 2015 , Erol et $\mathrm{al}^{28}$ reported outcomes of a retrospective review of 20 infants (36 eyes) with type 1 ROP who received either intravitreal bevacizumab (21 eyes; dose, $0.625 \mathrm{mg}$ ) or intravitreal ranibizumab (15 eyes; dose, 0.25 $\mathrm{mg})$. Recurrence was seen in 4 of $15(27 \%)$ eyes treated with ranibizumab and 2 of $21(10 \%)$ eyes treated with bevacizumab.

In 2017 , Kabatas et $\mathrm{al}^{29}$ reported outcomes of a retrospective review of 54 infants (108 eyes) with type 1 ROP who received intravitreal bevacizumab (24 eyes; dose, $0.625 \mathrm{mg}$ ), intravitreal ranibizumab (12 eyes; dose, 0.25 $\mathrm{mg}$ ), or diode photocoagulation (72 eyes). Main outcome measures assessed were recurrence of ROP, time to total retinal vascularization, and refractive errors. Recurrence was seen in 2 of $12(16 \%)$ eyes treated with ranibizumab and 2 of $24(8.3 \%)$ eyes treated with bevacizumab. There was no difference in refractive error among ranibizumab, bevacizumab, and laser photocoagulation groups. The mean time to complete vascularization in the bevacizumab group was $73 \pm 10.1$ weeks of PMA and $61.8 \pm 6.6$ weeks of PMA in the ranibizumab group.

In 2016, Lin et $\mathrm{al}^{30}$ reported outcomes of a retrospective review of 21 infants (40 eyes) with type 1 ROP who received either intravitreal bevacizumab (25 eyes; dose, $0.625 \mathrm{mg}$ ) or intravitreal ranibizumab (15 eyes; dose, 0.25 $\mathrm{mg})$. Main outcome measures were refractive status including axial length and refraction at a corrected age of 1 year. Complete vascularization was noted in 15 of 25 $(60 \%)$ eyes treated with ranibizumab and 7 of $15(47 \%)$ eyes treated with bevacizumab. There were no differences in the rates of axial length or spherical equivalent between ranibizumab and bevacizumab treatment groups.

In 2015, Wong et $\mathrm{al}^{19}$ reported outcomes of a retrospective review of 6 infants (10 eyes) with zone I or posterior zone II ROP who received either intravitreal bevacizumab (4 eyes; dose, $0.625 \mathrm{mg}$ ) or intravitreal ranibizumab (6 eyes; dose, $0.25 \mathrm{mg}$ ). Recurrence was seen in 5 of $6(83 \%)$ eyes treated with ranibizumab on average 5.9 weeks after treatment. No recurrence was detected in the four eyes treated with bevacizumab. One infant who 
received an unilateral injection of ranibizumab demonstrated bilateral regression of ROP.

In 2013, Castellanos et $\mathrm{al}^{31}$ reported outcomes of a retrospective review of 3 infants ( 6 eyes) with type 1 ROP treated with intravitreal ranibizumab $(0.25 \mathrm{mg})$ and noted complete resolution of neovascularization after single injection. Three year follow-up showed no evidence of recurrence or unfavorable structural outcomes.

In 2018, Tong et a ${ }^{34}$ reported outcomes of a retrospective review of 83 infants (160 eyes) with aggressive posterior ROP (APROP) treated with intravitreal ranibizumab $(0.25 \mathrm{mg})$. They noted that 35 of $160(22 \%)$ eyes progressed to retinal detachment and reported older postmenstrual age and low neutrophil count as independent risk factors for retinal detachment in APROP on multivariate analysis. Recurrence requiring retreatment occurred in 82 of 160 (51\%) eyes at a mean interval of $7.5 \pm 6.9$ weeks after the first intravitreal ranibizumab treatment.

In 2017 , Feng et $\mathrm{al}^{33}$ reported outcomes of a retrospective review of 331 infants (629 eyes) with APROP (105 eyes), type 1 ROP (411 eyes), and type 1 prethreshold ROP (113 eyes) treated with intravitreal ranibizumab $(0.25 \mathrm{mg})$. Recurrence was seen in 70 of $105(67 \%)$ eyes with APROP, 157 of 411 (38\%) eyes with type 1 ROP, and 18 of 113 (16\%) eyes with prethreshold ROP. Mean time to recurrence was $8.57 \pm 3.73$ weeks (range: 4-29 weeks) after treatment. The rate of recurrence was significantly higher in patients with zone I ROP (61\%, 101 of 164 eyes) than in zone II ROP $(31 \%, 144$ of 465 eyes). In patients with recurrence, additional treatments included a second intravitreal ranibizumab injection (92 eyes, 38\%), supplemental diode photocoagulation (146 eyes, 60\%), external scleral buckle ( 2 eyes, $0.8 \%$ ), and vitrectomy ( 5 eyes, $2 \%$ ).

In 2017 , Huang et $\mathrm{al}^{32}$ reported outcomes of a retrospective review of 145 infants (283 eyes) with type 1 ROP who were treated with intravitreal ranibizumab $(0.25 \mathrm{mg})$. All eyes were classified into 2 groups: positive response (regression of plus disease after injection, and/or retinal vessels continued to develop into the peripheral area) and negative/no response (defined as ROP worsened after injection and developed into Stage 4A, 4B, or 5, or plus disease and ridge did not show any change 1 week after injection). A total of 266 of 283 (94\%) eyes had a positive response after intravitreal ranibizumab, and of them, 127 eyes (45\%) had initial regression with subsequent recurrence. The recurrence rate was $47 \%$ in APROP, $58 \%$ in zone I, and $35 \%$ in zone II. The time between recurrence and initial treatment was $8.3 \pm 2.7$ weeks (range 2.3-15.4 weeks).

\section{Optimal dose of ranibizumab}

Ranibizumab is commercially available in a vial and prefilled syringe form in two concentrations: $0.5 \mathrm{mg} / 0.05 \mathrm{~mL}$ and $0.3 \mathrm{mg} / 0.05 \mathrm{~mL}$. To date, large multicenter trials found that the $0.5 \mathrm{mg}$ dose was effective to treat agerelated macular degeneration, whereas the $0.3 \mathrm{mg}$ dose was equally efficacious to treat diabetic macular edema and diabetic retinopathy. ${ }^{35,36}$ The findings and variable dosing intervals in the adult population support the premise that the dose of anti-VEGF drug may be disease specific or patient specific. Retinal neovascularization appears to be extremely sensitive to anti-VEGF therapy, and potentially lower anti-VEGF doses may be effective for ROP. However, the optimal dosages for both ranibizumab and bevacizumab remain unknown and controversial. A dose that results in effective regression of ROP while minimizing systemic penetration would be ideal due to uncertainties regarding how these drugs affect premature infants during neurodevelopmental growth.

The BEAT-ROP study demonstrated that $50 \%$ of the adult anti-VEGF dose of bevacizumab is effective in halting ROP progression in the large majority of infants. ${ }^{5}$ Therefore, in the literature, the majority of ROP cases treated with intravitreal ranibizumab use $50 \%(0.25 \mathrm{mg})$ of the adult dose (range, $0.1^{37}-0.3 \mathrm{mg}^{24}$ ). However, the best possible dose is unknown, and it is unclear if even lower doses of ranibizumab could be used with similar effectiveness. The neonate eye is estimated to be less than one-third the normal volume of an adult eye and, in particular, the vitreous volume comprises only $20 \%$ of an adult eye, which suggests that lower doses of anti-VEGF may be more appropriate. ${ }^{38,39}$

In the multicenter randomized CARE-ROP study, Stahl et al $^{18}$ compared 2 doses of ranibizumab (0.12 $\mathrm{mg}$ and $0.20 \mathrm{mg}$ ) in infants with bilateral ROP and assessed the number of infants who required rescue therapy at 24 weeks. A total of 14 of $16(88 \%)$ infants achieved control of ROP without the need for rescue therapy. Four infants ( 2 in each dose group) showed recurrence of ROP and required retreatment with ranibizumab. Another study of 24 eyes with type 1 prethreshold ROP who received $0.1 \mathrm{mg}$ of intravitreal ranibizumab showed regression of disease in all cases without any recurrence of disease or need for treatment at 54 weeks post-menstrual age. ${ }^{37}$ These findings coincide with dose de-escalation studies of bevacizumab for ROP, which found that dosing between $2.5 \%$ and $20 \%$ 
of the adult dose of bevacizumab may be effective in controlling acute ROP though these dose levels may lead to higher rates of recurrence. ${ }^{40,41}$

Effective dosing of ranibizumab may not only be related to the total dose of medication administered but also the timing of administration during the ROP disease course. The optimal window for treatment with anti-VEGF therapy is at the first sign of plus disease or neovascularization but before the formation of extensive fibrovascular membranes. When administered in late stage 4 or 5 disease, ranibizumab may cause contraction of the fibrovascular membranes and posterior hyaloid, thereby worsening tractional retinal detachment. ${ }^{42}$ These changes may be analogous to the "crunch" phenomenon observed in patients with proliferative diabetic retinopathy in which anti-VEGF therapy may worsen traction on the retina. ${ }^{43}$

\section{Risk of recurrence of ROP treated with ranibizumab}

The rates and timing of ROP recurrence are variable among study populations. As noted in Table 2, infants receiving intravitreal ranibizumab had a mean (range) rate of ROP recurrence of $41.1 \%$ (range, $0 \%$ to $83 \%$ ). The wide discrepancies in ROP recurrence may be due to the differences in the clinical definition of recurrence. In the studies examined, recurrence was variably defined as the reappearance of neovascularization, recurrent plus disease, extraretinal fibrovascular proliferation, appearance of a ridge, or progression of disease despite prior treatment. Additionally, dosing, treatment, zones, stages, and duration of follow-up vary, making direct comparison between series difficult. Furthermore, recurrences may also occur with laser monotherapy as ETROP reported unfavorable structural outcomes in $9 \%$ of infants undergoing early treatment. ${ }^{2}$

In comparison with intravitreal bevacizumab and conventional laser ablative therapy, recurrence after intravitreal ranibizumab has been observed more frequently than either intravitreal bevacizumab or laser monotherapy (Figure 2). Because ranibizumab is an antibody fragment with a shorter half-life, it is possible that the rate of recurrence after initial injection may be higher in eyes treated with ranibizumab because it is more rapidly cleared from the eye compared to bevacizumab. ${ }^{10}$ Gunay et $\mathrm{al}^{25}$ found a higher rate of ROP recurrence in eyes treated with ranibizumab (50\%) compared with bevacizumab (5.5\%), but noted no difference in retreatment rates. At 1 year of age, Chen et $\mathrm{al}^{27}$ reported that in a series of 72 eyes treated with intravitreal bevacizumab (41 eyes) or ranibizumab (31 eyes) there was no recurrence of ROP in either group when there was an initial response to treatment.

Most published studies do not stratify risk of ROP recurrence based on the initial classification of ROP disease. However, Feng et $\mathrm{al}^{33}$ specifically reported rates of ROP recurrence after intravitreal ranibizumab based on initial ROP classification and found that more aggressive forms of ROP at initial treatment were significantly correlated with ROP recurrence. Based on ROP classification, recurrence was seen in 70 of 105 (67\%) eyes with APROP, 157 of 411 (38\%) eyes with type 1 ROP, and 18 of 113 (16\%) eyes with prethreshold ROP. Higher rates of recurrence in eyes with APROP have previously been noted in patients receiving either intravitreal ranibizumab ${ }^{34}$ or bevacizumab ${ }^{44}$ with low

Rates of ROP recurrence after treatment with intravitreal ranibizumab versus bevacizumab

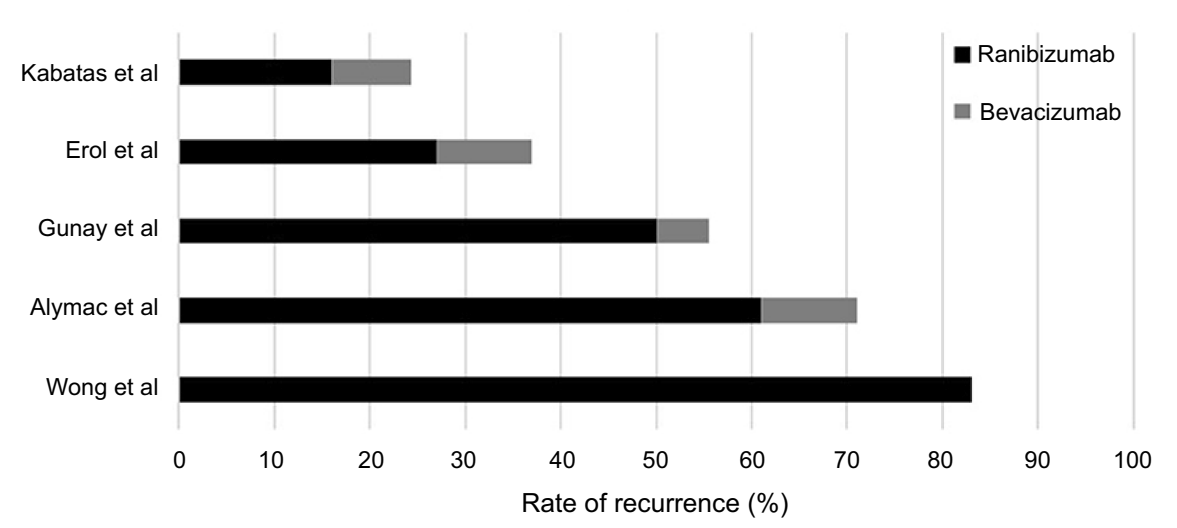

Figure 2 Rates of retinopathy of prematurity recurrence after treatment with intravitreal ranibizumab versus bevacizumab. Among the different studies, rates of recurrence in patients receiving intravitreal ranibizumab (black) ranged from $16 \%$ to $83 \%$, whereas rates of recurrence in patients receiving intravitreal bevacizumab (gray) ranged from $0 \%$ to $10 \%$. 
birthweight as a common risk factor for APROP recurrence and retreatment. Feng et $\mathrm{al}^{33}$ also noted that the rate of recurrence was significantly higher in patients with zone I ROP $(61 \%, 101$ of 164 eyes) than in zone II ROP $(31 \%, 144$ of 465 eyes). Another study of 283 eyes with type 1 ROP also showed recurrence rates in zone I ROP (58\%) were higher than that in zone II (35.4\%). ${ }^{32}$ The higher rates of recurrence in zone I ROP compared to zone II ROP may be because more time is needed to achieve full vascularization, which may increase the likelihood of subsequent rises on VEGF levels.

In the original BEAT-ROP study, time to recurrence was 19.2 weeks after intravitreal bevacizumab compared with 6.4 weeks after laser treatment for zone I disease. ${ }^{5}$ In comparison to recurrence after treatment with intravitreal bevacizumab, recurrence after treatment with intravitreal ranibizumab has been observed to occur earlier. ${ }^{25,28,33}$ Gunay et $\mathrm{al}^{25}$ showed, in 264 eyes with type 1 ROP, recurrence after treatment with ranibizumab occurred at a mean interval of $8.75 \pm 1.5$ weeks compared to $14 \pm 2.65$ weeks with bevacizumab. In 629 eyes with ROP treated with ranibizumab, Feng et $\mathrm{al}^{33}$ reported recurrence after initial treatment occurred at a mean interval of $8.57 \pm 3.73$ weeks (range: 4-29 weeks).

The higher frequency of recurrence after ranibizumab may be related to its shorter half-life and is an important consideration when balancing ranibizumab's shorter duration of systemic VEGF level suppression with its need for frequent follow-up and potential retreatment.

\section{Current trials: RAINBOW}

The phase III multicenter trial, RAnibizumab Compared With Laser Therapy for the Treatment of INfants BOrn Prematurely With Retinopathy of Prematurity (RAINBOW), has closed recruitment and will evaluate the efficacy and safety of ranibizumab compared with laser therapy in infants with ROP. To date, the RAINBOW trial (information available at: https:// clinicaltrials.gov/ct2/show/NCT02375971) is the first reported randomized clinical trial to evaluate the potential use of ranibizumab in ROP. Inclusion criteria for the study were 1) preterm infants with birth weight $<1500$ grams and 2) bilateral ROP with one of the following retinal findings in each eye: Zone I, stage $1+, 2+, 3$, or $3+$ disease; zone II, stage $3+$ disease; or APROP. 225 preterm infants were enrolled and randomized $1: 1: 1$ to receive in both eyes either intravitreal ranibizumab 0.2 $\mathrm{mg}$, intravitreal ranibizumab $0.1 \mathrm{mg}$, or laser photocoagulation therapy. The primary outcome measure was the percentage of patients with absence of active ROP at 24 weeks, no intervention with a second modality for ROP (treatment switch) until 24 weeks, and absence of unfavorable structural outcomes in both eyes at or before 24 weeks. Unfavorable structural outcomes included retrolental membrane, substantial temporal retinal vessel dragging/macular ectopia posterior retinal fold involving the macula, and retinal detachment involving the macula. Limitations of this study include a large number of sites with low recruitment per site, and many international sites with a large proportion of Asian patients.

In addition, the RAINBOW trial had a variety of secondary outcomes to assess the potential systemic effects of ranibizumab in this study population. In particular, the mean change in serum ranibizumab concentration and mean change in serum VEGF levels were assessed at pre-specified time intervals before and after treatment. Furthermore, neurodevelopmental vital signs including body length, weight, blood pressure, head circumference, and knee to heel length were collected at prespecified time points in the study. Long-term safety and efficacy data will be collected until participants are 5 years of age, and will be investigated in the RAINBOW Extension study.

\section{Summary}

With the advancement of neonatal care to save more premature infants, particularly those with earlier gestational age and lower birthweights, ROP continues to be a significant cause of visual morbidity worldwide. Laser photocoagulation has been the previous standard of care for treatment-requiring ROP; however, intravitreal antiVEGF therapy is now another treatment option with increasing evidence supporting its use. Anti-VEGF therapy has proven effective in inducing acute regression of ROP, but concerns regarding safety, dosing, and recurrence remain. Ranibizumab is of particular interest because its vitreous half-life approaches that of bevacizumab, but after reaching systemic circulation, the elimination half-life is a few hours rather than weeks potentially resulting in a less effect on serum VEGF levels. Although this could be a distinct advantage for the treatment of ROP, continued research, including the results of the RAINBOW randomized control trial, are needed to determine the optimal dose of ranibizumab in ROP, recurrence rate and timing with need for retreatment, long-term ocular outcomes, and long-term systemic side effects.

\section{Disclosure}

MAK: speaker \& consultant (Genentech), consultant (Novartis) and consultant (Allergan). MAK reports personal 
fees from Genentech, Novartis and Allergan, outside the submitted work. The authors report no other conflicts of interest in this work.

\section{References}

1. Cryotherapy for retinopathy of prematurity cooperative group. Multicenter trial of cryotherapy for retinopathy of prematurity: preliminary results. Pediatrics. 1988;81(5):697-706.

2. Early treatment for retinopathy of prematurity cooperative group. Revised indications for the treatment of retinopathy of prematurity: results of the early treatment for retinopathy of prematurity randomized trial. Arch Ophthalmol. 2003;121(12):1684-1694.

3. VanderVeen DK, Melia M, Yang MB, Hutchinson AK, Wilson LB, Lambert SR. Anti-vascular endothelial growth factor therapy for primary treatment of type 1 retinopathy of prematurity: a report by the american academy of ophthalmology. Ophthalmology. 2017;124 (5):619-633

4. Geloneck MM, Chuang AZ, Clark WL, et al. Refractive outcomes following bevacizumab monotherapy compared with conventional laser treatment: a randomized clinical trial. JAMA Ophthalmol. 2014;132(11):1327-1333.

5. Mintz-Hittner HA, Kennedy KA, Chuang AZ. Efficacy of intravitreal bevacizumab for stage $3+$ retinopathy of prematurity. $N$ Engl J Med. 2011;364(7):603-615.

6. Zou L, Lai H, Zhou Q, Xiao F, Haigh JJ. Lasting controversy on ranibizumab and bevacizumab. Theranostics. 2011;1:395-402.

7. Hoeben A, Landuyt B, Highley MS, Wildiers H, Van Oosterom AT, De Bruijn EA. Vascular endothelial growth factor and angiogenesis. Pharmacol Rev. 2004;56(4):549-580.

8. Levy AP, Levy NS, Loscalzo J, et al. Regulation of vascular endothelial growth factor in cardiac myocytes. Circ Res. 1995;76(5):758766.

9. Bakri SJ, Snyder MR, Reid JM, Pulido JS, Ezzat MK, Singh RJ. Pharmacokinetics of intravitreal ranibizumab (lucentis). Ophthalmology. 2007;114(12):2179-2182.

10. Avery RL, Castellarin AA, Steinle NC, et al. Systemic pharmacokinetics and pharmacodynamics of intravitreal aflibercept, bevacizumab, and ranibizumab. Retina. 2017;37(10):1847-1858. doi:10.1097/ IAE.0000000000001493

11. Tawse KL, Jeng-Miller KW, Baumal CR. Current practice patterns for treatment of retinopathy of prematurity. Ophthalmic Surg Lasers Imaging Retina. 2016;47(5):491-495. doi:10.3928/23258160-20160419-16

12. Haigh JJ. Role of vegf in organogenesis. Organogenesis. 2008;4 (4):247-256. doi:10.4161/org.4.4.7415

13. Carneiro AM, Costa R, Falcao MS, et al. Vascular endothelial growth factor plasma levels before and after treatment of neovascular age-related macular degeneration with bevacizumab or ranibizumab. Acta Ophthalmol.2012;90(1):e25-e30. doi:10.1111/j.1755-3768.2011.02240.x

14. Sato T, Wada K, Arahori H, et al. Serum concentrations of bevacizumab (avastin) and vascular endothelial growth factor in infants with retinopathy of prematurity. Am J Ophthalmol. 2012;153 (2):327-333.e321. doi:10.1016/j.ajo.2011.07.005

15. Kong L, Bhatt AR, Demny AB, et al. Pharmacokinetics of bevacizumab and its effects on serum vegf and igf- 1 in infants with retinopathy of prematurity. Invest Ophthalmol Vis Sci. 2015;56(2):956-961. doi:10.1167/iovs.14-15842

16. Wu WC, Lien R, Liao PJ, et al. Serum levels of vascular endothelial growth factor and related factors after intravitreous bevacizumab injection for retinopathy of prematurity. JAMA Ophthalmol. 2015;133(4):391-397. doi:10.1001/jamaophthalmol.2014.5373

17. Zhou Y, Jiang Y, Bai Y, Wen J, Chen L. Vascular endothelial growth factor plasma levels before and after treatment of retinopathy of prematurity with ranibizumab. Graefes Arch Clin Exp Ophthalmol. 2016;254(1):31-6. doi:10.1007/s00417-015-2996-0
18. Stahl A, Krohne TU, Eter N, et al. Comparing alternative ranibizumab dosages for safety and efficacy in retinopathy of prematurity: a randomized clinical trial. JAMA Pediatr. 2018;172(3):278-286. doi:10.1001/jamapediatrics.2017.4838

19. Wong RK, Hubschman S, Tsui I. Reactivation of retinopathy of prematurity after ranibizumab treatment. Retina. 2015;35(4):675680. doi:10.1097/IAE.0000000000000578

20. Morin J, Luu TM, Superstein R, et al. Neurodevelopmental outcomes following bevacizumab injections for retinopathy of prematurity. Pediatrics. 2016;137:4. doi:10.1542/peds.2015-3218

21. Araz-Ersan B, Kir N, Tuncer S, et al. Preliminary anatomical and neurodevelopmental outcomes of intravitreal bevacizumab as adjunctive treatment for retinopathy of prematurity. Curr Eye Res. 2015;40 (6):585-591. doi:10.3109/02713683.2014.941070

22. Lien R, Yu M-H, Hsu K-H, et al. Neurodevelopmental outcomes in infants with retinopathy of prematurity and bevacizumab treatment. PLoS ONE. 2016;11(1):e0148019-e0148019. doi:10.1371/journal. pone.0148019

23. Sankar MJ, Sankar J, Chandra P. Anti-vascular endothelial growth factor (vegf) drugs for treatment of retinopathy of prematurity. Cochrane Database Syst Rev. 2018;1:Cd009734.

24. Zhang G, Yang M, Zeng J, et al. Comparison of intravitreal injection of ranibizumab versus laser therapy for zone ii treatment-requiring retinopathy of prematurity. Retina. 2017;37(4):710-717. doi:10.1097/ IAE.0000000000001241

25. Gunay M, Sukgen EA, Celik G, Kocluk Y. Comparison of bevacizumab, ranibizumab, and laser photocoagulation in the treatment of retinopathy of prematurity in turkey. Curr Eye Res. 2017;42 (3):462-469. doi:10.1080/02713683.2016.1196709

26. Alyamac Sukgen E, Comez A, Kocluk Y, Cevher S. The process of retinal vascularization after anti-vegf treatment in retinopathy of prematurity: a comparison study between ranibizumab and bevacizumab. Ophthalmologica. 2016;236(3):139-147. doi:10.1159/000449 530

27. Chen SN, Lian I, Hwang YC, et al. Intravitreal anti-vascular endothelial growth factor treatment for retinopathy of prematurity: comparison between ranibizumab and bevacizumab. Retina. 2015;35 (4):667-674. doi:10.1097/IAE.0000000000000380

28. Erol MK, Coban DT, Sari ES, et al. Comparison of intravitreal ranibizumab and bevacizumab treatment for retinopathy of prematurity. Arq Bras Oftalmol. 2015;78(6):340-343. doi:10.5935/00042749.20150090

29. Kabatas EU, Kurtul BE, Altiaylik Ozer P, Kabatas N. Comparison of intravitreal bevacizumab, intravitreal ranibizumab and laser photocoagulation for treatment of type 1 retinopathy of prematurity in turkish preterm children. Curr Eye Res. 2017;42(7):1054-1058. doi:10.1080/02713683.2016.1264607

30. Lin CJ, Tsai YY. Axial length, refraction, and retinal vascularization 1 year after ranibizumab or bevacizumab treatment for retinopathy of prematurity. Clin Ophthalmol. 2016;10:1323-1327. doi:10.2147/ OPTH.S110717

31. Castellanos MA, Schwartz S, Garcia-Aguirre G, Quiroz-Mercado H. Short-term outcome after intravitreal ranibizumab injections for the treatment of retinopathy of prematurity. Br J Ophthalmol. 2013;97 (7):816-819. doi:10.1136/bjophthalmol-2012-302276

32. Huang Q, Zhang Q, Fei P, et al. Ranibizumab injection as primary treatment in patients with retinopathy of prematurity: anatomic outcomes and influencing factors. Ophthalmology. 2017;124(8):11561164. doi:10.1016/j.ophtha.2017.03.018

33. Feng J, Qian J, Jiang Y, et al. Efficacy of primary intravitreal ranibizumab for retinopathy of prematurity in china. Ophthalmology. 2017;124(3):408-409. doi:10.1016/j.ophtha.2016.10.032

34. Tong Q, Yin H, Zhao M, Li X, Yu W. Outcomes and prognostic factors for aggressive posterior retinopathy of prematurity following initial treatment with intravitreal ranibizumab. BMC Ophthalmol. 2018;18(1):150. doi:10.1186/s12886-018-0815-1 
35. Brown DM, Nguyen QD, Marcus DM, et al. Long-term outcomes of ranibizumab therapy for diabetic macular edema: the 36-month results from two phase iii trials: rise and ride. Ophthalmology. 2013;120(10):2013-2022. doi:10.1016/j.ophtha.2013.02.034

36. Rosenfeld PJ, Brown DM, Heier JS, et al. Ranibizumab for neovascular age-related macular degeneration. $N$ Engl J Med. 2006;355 (14):1419-1431. doi:10.1056/NEJMoa054481

37. Ahmed IS, Hadi AM, Hassan HH. Efficacy of ultra-low-dose ( 0.1 $\mathrm{mg}$ ) ranibizumab intravitreal injection for treatment of prethreshold type 1 retinopathy of prematurity: a case series. Eur J Ophthalmol. Epub 2018 Nov 15.

38. Tasman W. Retinopathy of prematurity: do we still have a problem?: the charles 1. Schepens lecture. Arch Ophthalmol. 2011;129(8):10831086. doi:10.1001/archophthalmol.2011.192

39. Lemley CA, Han DP. An age-based method for planning sclerotomy placement during pediatric vitrectomy: a 12-year experience. Trans Am Ophthalmol Soc. 2007;105:86-89. discussion 89-91.

40. Wallace DK, Dean TW, Hartnett ME, et al. A dosing study of bevacizumab for retinopathy of prematurity: late recurrences and additional treatments. Ophthalmology. 2018;125(12):1961-1966. doi:10.1016/j. ophtha.2018.05.001
41. Wallace DK, Kraker RT, Freedman SF, et al. Assessment of lower doses of intravitreous bevacizumab for retinopathy of prematurity: a phase 1 dosing study. JAMA Ophthalmol. 2017;135(6):654-656. doi:10.1001/jamaophthalmol.2017.1055

42. Yonekawa $\mathrm{Y}, \mathrm{Wu} \mathrm{WC}$, Nitulescu $\mathrm{CE}$, et al. Progressive retinal detachment in infants with retinopathy of prematurity treated with intravitreal bevacizumab or ranibizumab. Retina. 2018;38(6):10791083. doi:10.1097/IAE.0000000000001685

43. Arevalo JF, Maia M, Flynn HW Jr, et al. Tractional retinal detachment following intravitreal bevacizumab (avastin) in patients with severe proliferative diabetic retinopathy. Br J Ophthalmol. 2008;92 (2):213-216. doi:10.1136/bjo.2007.127142

44. Mintz-Hittner HA, Geloneck MM, Chuang AZ. Clinical management of recurrent retinopathy of prematurity after intravitreal bevacizumab monotherapy. Ophthalmology. 2016;123(9):1845-1855. doi:10.1016/ j.ophtha.2016.04.028
Eye and Brain

\section{Publish your work in this journal}

Eye and Brain is an international, peer-reviewed, open access journal focusing on clinical and experimental research in the field of neuroophthalmology. All aspects of patient care are addressed within the journal as well as basic research. Papers covering original research, basic science, clinical and epidemiological studies, reviews and

Submit your manuscript here: https://www.dovepress.com/eye-and-brain-journal
Dovepress

evaluations, guidelines, expert opinion and commentary, case reports and extended reports are welcome. The manuscript management system is completely online and includes a very quick and fair peerreview system, which is all easy to use. Visit http://www.dovepress. com/testimonials.php to read real quotes from published authors. 